\title{
Obstructive jaundice caused by intraductal metastasis of lung adenocarcinoma
}

This article was published in the following Dove Press journal:

OncoTargets and Therapy

7 October 2014

Number of times this article has been viewed

\author{
Nobuaki Ochi' \\ Daisuke Goto ${ }^{2}$ \\ Hiromichi Yamane' \\ Tomoko Yamagishi' \\ Yoshihiro Honda' \\ Yasumasa Monobe 3 \\ Hirofumi Kawamoto ${ }^{2}$ \\ Nagio Takigawa' \\ 'Department of General Internal \\ Medicine $4,{ }^{2}$ Department of General \\ Internal Medicine 2, ${ }^{3}$ Department \\ of Pathology, Kawasaki Hospital, \\ Kawasaki Medical School, \\ Okayama, Japan
}

Correspondence: Nagio Takigawa Department of General Internal Medicine 4, Kawasaki Medical School, 2-I-80, Nakasange, Kita-ku, Okayama, 700-8505, Japan

Tel +8I 8622521 II

Fax +8I 862328343

Email ntakigaw@gmail.com

\begin{abstract}
Obstructive jaundice caused by metastases to the porta hepatis is often observed in patients with various advanced cancers; however, metastasis of lung cancer to the common bile duct with subsequent development of jaundice is rare. A 75-year-old female with lung adenocarcinoma harboring epidermal growth factor receptor (EGFR) mutation (15-bp in-frame deletion in exon 19 and T790M in exon 20) developed obstructive jaundice during therapy. Obstruction of the common bile duct caused by an intraductal tumor was identified by computed tomography, endoscopic retrograde cholangiopancreatography, and endoscopic ultrasonography. Although primary cholangiocarcinoma was highly suspected according to the imaging findings, immunohistochemical evaluation of the intraductal tumor demonstrated thyroid transcription factor-1 positive adenocarcinoma. Furthermore, peptide nucleic acid-locked nucleic acid polymerase chain reaction clamp analysis showed that the tumor contained the same EGFR mutation as that in the primary lung cancer. Thus, we confirmed intraductal metastasis from a lung adenocarcinoma. To our knowledge, this is the second report of obstructive jaundice caused by intraductal metastasis of lung cancer.
\end{abstract}

Keywords: lung cancer, cholangiocarcinoma, EGFR

\section{Introduction}

Cholangiocarcinoma is the second most common primary liver tumor worldwide after hepatocellular carcinoma. ${ }^{1}$ It arises from the epithelium of the bile duct and is difficult to diagnose because of its late clinical manifestation. The presentation of cholangiocarcinoma depends on the location of the tumor. When the tumor is located at the bifurcation of the hepatic ducts or the distal common bile duct, patients frequently exhibit obstructive jaundice, pale stools, and bilirubinuria. Meanwhile, obstructive jaundice is observed in patients with various advanced cancers metastasizing to the hepatic portal region. The primary tumors most commonly associated with the development of obstructive jaundice are primary tumors of the stomach, colon, and breast. ${ }^{2}$ Lung cancer accounts for less than $1 \%$ of these tumors, and small-cell lung cancer has been reported in the literature..$^{2-6}$ The most common extrathoracic metastatic sites in autopsied patients with non-small-cell lung cancer were liver (31\%-47\%), brain (34\%-35\%), bone $(29 \%-34 \%)$, and adrenal gland $(29 \%-31 \%){ }^{7,8}$ Thus, obstructive jaundice by direct metastasis to the common bile duct seems to be a rare manifestation of lung cancer.

We herein describe a patient with lung cancer who developed obstructive jaundice due to metastasis to the common bile duct. The patient's condition mimicked primary cholangiocarcinoma on diagnostic images. Immunohistochemistry and epidermal 
growth factor receptor (EGFR) mutation analysis proved that the intraductal tumor was a metastasis from the lung adenocarcinoma.

\section{Case report}

A 75-year-old female presented with dyspnea. She was an exsmoker (60 pack-years). Her Eastern Cooperative Oncology Group performance status was 1. Chest computed tomography (CT) revealed a pulmonary mass in the right upper lobe with right-sided pleural effusion. She was diagnosed with advanced lung adenocarcinoma (cT2aN3M1a, stage IV) by transbronchial biopsy and imaging findings. Because peptide nucleic acid-locked nucleic acid (PNA-LNA) polymerase chain reaction (PCR) clamp analysis showed that the primary lung tumor had an EGFR mutation (15-bp in-frame deletion in exon 19, del E746-A750), she was treated with gefitinib as first-line therapy for 14 months. However, her disease progressed, and thereafter she received six cycles of carboplatin and pemetrexed chemotherapy followed by pemetrexed for maintenance. During the maintenance therapy, she developed nausea and anorexia and had a performance status of 3 . Imaging studies showed that her right-sided malignant pleural effusion had increased in severity and that multiple brain metastases and meningitis carcinomatosa had developed. T790M EGFR-tyrosine kinase inhibitor-resistant mutation in exon 20, in addition to the EGFR exon 19 deletion mutation, was identified from the pleural effusion. Adenocarcinoma cells were also observed in her spinal fluid. PNA-LNA PCR clamp analysis of the spinal fluid revealed the same EGFR deletion mutation; however, the T790M mutation could not be detected. Thus, we administered erlotinib to control the central nervous system metastasis. After 4 weeks, the brain metastases responded completely and her digestive symptoms related to the central nervous system metastasis disappeared.

After receiving erlotinib for 3 months, the patient visited the emergency department with jaundice, epigastric pain, and a low-grade fever. Hematological investigation showed a hemoglobin level of $9.4 \mathrm{~g} / \mathrm{dL}$; her white blood cell count and differential leukocyte and platelet counts were within normal limits. Her serum C-reactive protein level was $1.76 \mathrm{mg} / \mathrm{dL}$. Her other laboratory findings were as follows: total bilirubin, $5.8 \mathrm{mg} / \mathrm{dL}$; direct bilirubin, $3.8 \mathrm{mg} / \mathrm{dL}$; aspartate aminotransferase, $165 \mathrm{U} / \mathrm{L}$; alanine aminotransferase, $111 \mathrm{U} / \mathrm{L}$; $\gamma$-glutamyl transpeptidase, $148 \mathrm{U} / \mathrm{L}$; lactate dehydrogenase, $223 \mathrm{U} / \mathrm{L}$; amylase, $44 \mathrm{U} / \mathrm{L}$; blood urea nitrogen, $23 \mathrm{mg} / \mathrm{dL}$; creatinine, $0.58 \mathrm{mg} / \mathrm{dL}$; prothrombin time, 11.3 seconds; activated partial thromboplastin time,
26.1 seconds; and D-dimers, $2.7 \mu \mathrm{g} / \mathrm{mL}$. A urinalysis revealed bilirubinuria $(1+)$.

Chest X-ray and $\mathrm{CT}$ revealed bilateral massive pleural effusion. Abdominal CT revealed dilatation of the intraand extrahepatic bile duct, including the common bile duct, which showed abrupt termination on the distal side (Figure 1A and B). No metastases to the porta hepatis, including any originating from the lymph nodes, liver, or pancreas, were observed. Computer-reconstructed coronal section images also confirmed the obstruction of the distal common bile duct, and an intraductal tumor in the dilated common bile duct with slightly low attenuation was present on CT (Figure 1C). Endoscopic retrograde cholangiopancreatography and endoscopic ultrasonography also showed obstruction of the common bile duct due to the intraductal tumor (Figure 1D and E). The appearance of the intraductal tumor was observed by peroral cholangioscopy. The surface of the common bile duct showed irregular and inadequate angiogenesis. Detailed observation was difficult due to the presence of stenosis. Based on these findings, we suspected primary cholangiocarcinoma. An endoscopic retrograde bile duct drainage tube was placed, and the patient's jaundice promptly improved. Adenocarcinoma cells in a specimen obtained by transbronchial biopsy at the time of diagnosis are shown in Figure 2A and B. The biopsy specimen obtained using digestive endoscopy revealed poorly differentiated adenocarcinoma that was positive for thyroid transcription factor-1 by immunohistochemistry (Figure 2C and D). PNA-LNA PCR clamp analysis revealed that the specimen had an EGFR exon 19 deletion mutation and T790M

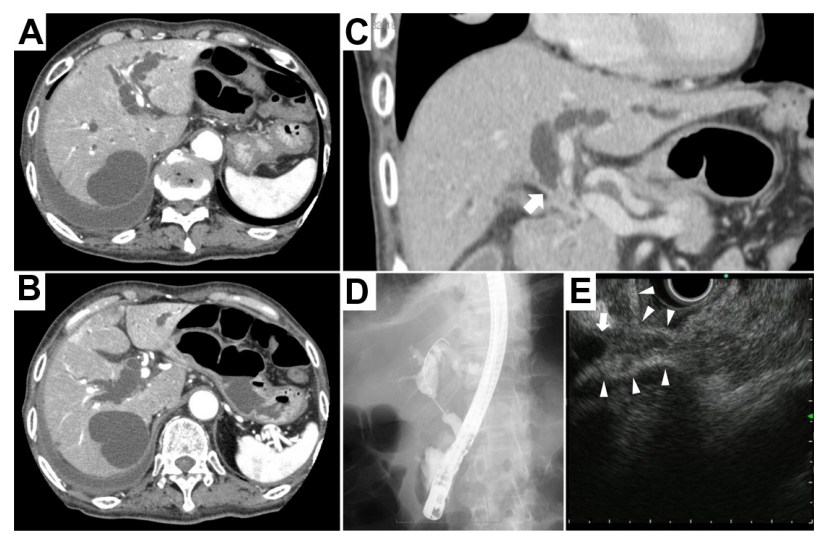

Figure I Radiological images.

Notes: (A and B) Contrast-enhanced abdominal computed tomography revealed dilatation of the intra- and extrahepatic bile duct, including the common bile duct. (C) A computer-reconstructed coronal section image revealed obstruction of the distal common bile duct, which showed abrupt termination on the distal side. (D and E) Endoscopic retrograde cholangiopancreatography and endoscopic ultrasound revealed obstruction of the common bile duct secondary to the intraductal tumor. The arrow indicates the distal end of the common bile duct. The arrowheads indicate the wall of the common bile duct and cystic duct. 


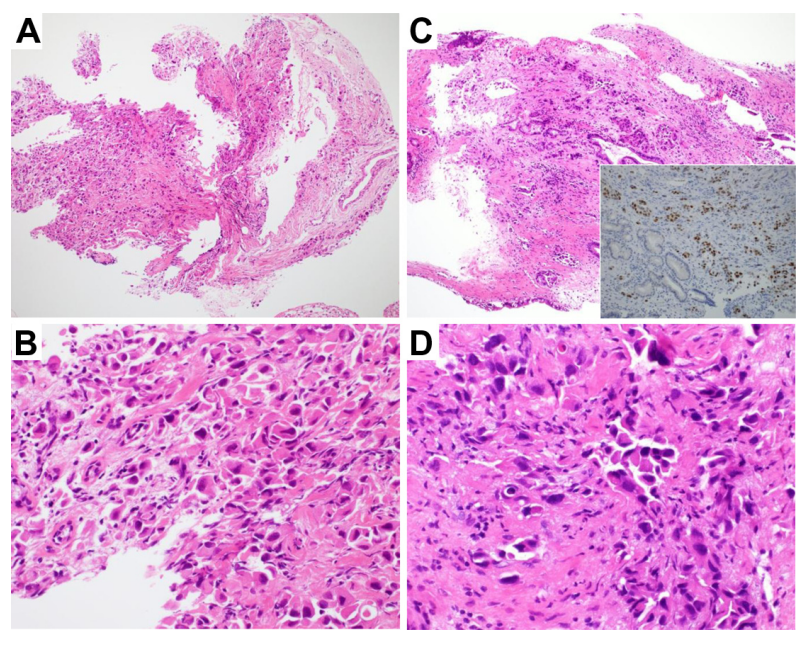

Figure 2 Pathological specimens and cholangioscopic images.

Notes: (A and B) Adenocarcinoma cells in a specimen obtained by transbronchial biopsy. (A) $\times 40$; (B) $\times 400$. (C and D) Adenocarcinoma cells in a specimen obtained from the intraductal tumor. $(C) \times 40$. Inset: thyroid transcription factor-I stain. (D) $\times 400$.

mutation in exon 20. Two weeks later, the patient received a replacement endoscopic retrograde bile duct drainage tube with a self-expanding metal stent.

\section{Discussion}

We observed obstructive jaundice caused by intraductal metastasis in a patient with adenocarcinoma of the lung. Immunohistochemistry and EGFR mutation analysis were useful to differentiate metastatic intraductal tumor from primary cholangiocarcinoma.

Imaging studies are essential for identifying the cause of biliary obstruction. Ultrasonography is a convenient approach to the detection of biliary tract dilatation; however, it is less effective for determination of the cause and extent of the obstruction. ${ }^{9,10} \mathrm{CT}$ evaluation was insufficient and ineffective in the assessment of resectability of parenchyma and biliary duct, and in the determination of portal involvement. Contrast-enhanced, triple-phase, helical CT and multiplanar reconstruction images have recently proved useful for detecting intrahepatic cholangiocarcinoma or longitudinal ductal spread of hilar cholangiocarcinoma. ${ }^{11-13}$ Although we did not perform magnetic resonance cholangiopancreatography, this procedure may have some advantages over $\mathrm{CT}$; its intrinsic high tissue contrast and multiplanar capability may increase diagnostic accuracy. ${ }^{14-18}$ The diagnostic images suggested that the lesion in the present case was most likely to be primary cholangiocarcinoma. The biopsy specimen of the intraductal tumor was histologically proven to be adenocarcinoma. Because more than $90 \%$ of cholangiocarcinomas are adenocarcinomas, ${ }^{19}$ pathological differentiation between primary cholangiocarcinoma and metastatic adenocarcinoma from other organs using hematoxylin-eosin staining alone is difficult.

We also examined the protein expression and EGFR gene mutation status in the present case. Immunohistochemical staining showed that the adenocarcinoma cells were positive for thyroid transcription factor-1. PNA-LNA PCR clamp analysis demonstrated that the EGFR mutation status of the primary lung cancer was identical to that of the intraductal tumor. EGFR del E746-A750 and T790M mutations have not been reported in cholangiocarcinoma, although there have been reports of other EGFR mutations in exons 20 and 21..$^{20,21}$ Thus, the protein and gene analyses led to a definitive diagnosis of metastasis from lung cancer. Cha et al recently reported that immunohistochemical study of the biopsy sample from bile duct with thyroid transcription factor- 1 could be useful in differential diagnosis..$^{22}$ To our knowledge, this is the second report of metastasis of lung adenocarcinoma to the bile duct with subsequent development of obstructive jaundice.

\section{Conclusion}

Biliary obstruction in patients with lung adenocarcinoma might exhibit the typical patterns of primary cholangiocarcinoma on CT and endoscopic retrograde cholangiopancreatographic images. Clinicians should be aware that EGFR mutation analysis of metastatic lesions might lead to a definitive diagnosis.

\section{Author contributions}

All authors contributed toward data analysis, drafting and revising the paper and agree to be accountable for all aspects of the work.

\section{Disclosure}

Dr Nagio Takigawa received honoraria from AstraZeneca, Chugai Pharmaceutical, and Eli Lilly. The authors report no other conflicts of interest in this work.

\section{References}

1. Khan SA, Davidson BR, Goldin RD, et al; British Society of Gastroenterology. Guidelines for the diagnosis and treatment of cholangiocarcinoma: an update. Gut. 2012;61(12):1657-1669.

2. Lokich JJ, Kane RA, Harrison DA, McDermott WV. Biliary tract obstruction secondary to cancer: management guidelines and selected literature review. J Clin Oncol. 1987;5(6):969-981.

3. Ochi N, Takigawa N, Yasugi M, et al. Obstructive jaundice at the initial presentation in small-cell lung cancer. Int Med Case Rep J. 2010;3:9-12.

4. Johnson DH, Hainsworth JD, Greco FA. Extrahepatic biliary obstruction caused by small-cell lung cancer. Ann Intern Med. 1985;102(4):487-490. 
5. Dunkerley RC, Dunn GD. Use of retrograde cholangiography in guiding radiotherapy of obstructive jaundice due to tumor. Am J Gastroenterol. 1976;66(3):283-286.

6. Obara M, Satoh H, Yamashita YT, et al. Metastatic small cell lung cancer causing biliary obstruction. Med Oncol. 1998;15(4):292-294.

7. Stenbygaard LE, Sørensen JB, Larsen H, Dombernowsky P. Metastatic pattern in non-resectable non-small cell lung cancer. Acta Oncol. 1999;38(8):993-998.

8. Stenbygaard LE, Sørensen JB. Small bowel metastases in non-small cell lung cancer. Lung Cancer. 1999;26(2):95-101.

9. Vas W, Salem S. Accuracy of sonography and transhepatic chloangiography in obstructive jaundice. J Can Assoc Radiol. 1981;32(2):111-113.

10. Honickman SP, Mueller PR, Wittenberg J, et al. Ultrasound in obstructive jaundice: prospective evaluation of site and cause. Radiology. 1983;147(2):511-515.

11. Valls C, Gumà A, Puig I, et al. Intrahepatic peripheral cholangiocarcinoma: CT evaluation. Abdom Imaging. 2000;25(5):490-496.

12. Ajiki T, Fukumoto T, Ueno K, Okazaki T, Matsumoto I, Ku Y. Three-dimensional computed tomographic cholangiography as a novel diagnostic tool for evaluation of bile duct invasion of perihilar cholangiocarcinoma. Hepatogastroenterology. 2013;60(128): 1833-1838.

13. NagakawaY, Kasuya K, Bunso K, et al. Usefulness of multi-3-dimensional computed tomograms fused with multiplanar reconstruction images and peroral cholangioscopy findings in hilar cholangiocarcinoma. J Hepatobiliary Pancreat Sci. 2014;21(4):256-262.

14. Vaishali MD, Agarwal AK, Upadhyaya DN, Chauhan VS, Sharma OP, Shukla VK. Magnetic resonance cholangiopancreatography in obstructive jaundice. J Clin Gastroenterol. 2004;38(10):887-890.
15. Rahman R, Ju J, Shamma's J, Goebel S, Sundaram U. Correlation between MRCP and ERCP findings at a tertiary care hospital. WVMed J. 2010;106(5):14-19.

16. Manfredi R, Barbaro B, Masselli G, Vecchioli A, Marano P. Magnetic resonance imaging of cholangiocarcinoma. Semin Liver Dis. 2004;24(2): 155-164.

17. Masselli G, Manfredi R, Vecchioli A, Gualdi G. MR imaging and MR cholangiopancreatography in the preoperative evaluation of hilar cholangiocarcinoma: correlation with surgical and pathologic findings. Eur Radiol. 2008;18(10):2213-2221.

18. Liang C, Mao H, Wang Q, et al. Diagnostic performance of magnetic resonance cholangiopancreatography in malignant obstructive jaundice. Cell Biochem Biophys. 2011;61(2):383-388.

19. Ishak KG, Anthony PP, Sobin LH. Histological Typing of Tumours in the Liver. World Health Organization International Histological Classification of Tumours. 2nd ed. Berlin: Springer-Verlag; 1994.

20. Wheler JJ, Falchook GS, Tsimberidou AM, et al. Aberrations in the epidermal growth factor receptor gene in 958 patients with diverse advanced tumors: implications for therapy. Ann Oncol. 2013;24(3):838-842.

21. Chang YT, Chang MC, Huang KW, Tung CC, Hsu C, Wong JM. Clinicopathological and prognostic significances of EGFR, KRAS and BRAF mutations in biliary tract carcinomas in Taiwan. J Gastroenterol Hepatol. 2014;29(5):1119-1125.

22. Cha IH, Kim JN, Kim YS, Ryu SH, Moon JS, Lee HK. Metastatic common bile duct cancer from pulmonary adenocarcinoma presenting as obstructive jaundice. Korean J Gastroenterol. 2013;61(1):50-53.
OncoTargets and Therapy

\section{Publish your work in this journal}

OncoTargets and Therapy is an international, peer-reviewed, open access journal focusing on the pathological basis of all cancers, potential targets for therapy and treatment protocols employed to improve the management of cancer patients. The journal also focuses on the impact of management programs and new therapeutic agents and protocols on

\section{Dovepress}

patient perspectives such as quality of life, adherence and satisfaction. The manuscript management system is completely online and includes a very quick and fair peer-review system, which is all easy to use. Visit http://www.dovepress.com/testimonials.php to read real quotes from published authors. 